\title{
Assessment of Rarefied Hypersonic Aerodynamics Modeling and Windtunnel Data
}

\author{
Jose F. Padilla $^{1}$ and Iain D. Boyd ${ }^{2}$ \\ University of Michigan, Ann Arbor, MI, 48109
}

\begin{abstract}
The future of space exploration and development will be determined by our ability to safely access and return from space. Steady improvements in the prediction of atmospheric entry phase conditions have occurred through comparisons of results obtained in the windtunnel and from computer simulation. In this study, computer simulations are presented using the direct simulation Monte Carlo (DSMC) method for hypersonic rarefied flow and compared to existing windtunnel data. Modified Newtonian and free molecular flow methods are employed to compliment the DSMC aerodynamic analysis. There is close agreement between the DSMC and windtunnel data for a blunted-cone windtunnel model. The computed aerodynamic properties are largely insensitive to modest changes in accommodation coefficients. For ballistic capsule models, there is significantly greater disagreement between the DSMC and windtunnel data. The DSMC numerical procedures are verified with results from an independent code and the windtunnel data are therefore considered suspect.
\end{abstract}

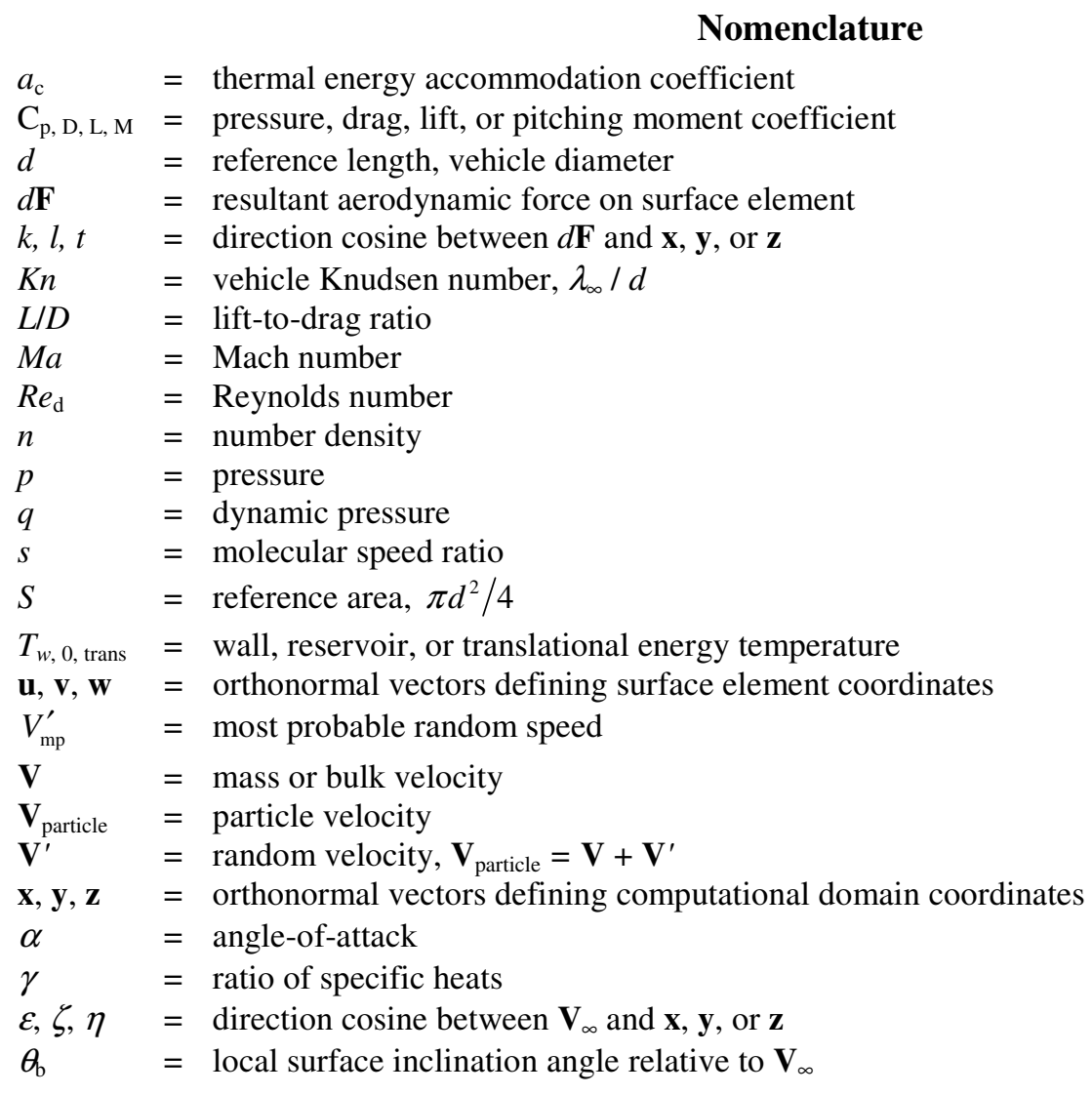

\footnotetext{
${ }^{1}$ Graduate Student, Department of Aerospace Engineering, Student Member AIAA.

${ }^{2}$ Professor, Department of Aerospace Engineering, Associate Fellow AIAA.
} 


$\begin{array}{ll}\rho & =\text { mass density } \\ \tau & =\text { shear stress } \\ \sigma_{\mathrm{n}, \mathrm{t}} & =\text { normal or tangential reflection coefficient }\end{array}$

Subscripts

$\infty \quad=$ of the free-stream

$\mathrm{i}, \mathrm{r}=$ of particle incident to, or reflected from, a surface

$\mathrm{M}(\cdot) \quad=$ pitching moment about point $(\cdot)$

$u, v=$ component along $u$ or $v$ local surface coordinate

$x, y, z=$ component along $x, y$, or $z$ global domain coordinate

\section{Introduction}

$\mathrm{T}$ HE entering of a planetary atmosphere at a near-orbital velocity has been a common scene in space exploration. This transportation phase is expected to become more and more common with the resurgence of manned space exploration. In space exploration, NASA has decided to make it a goal to send people back to the Moon and then onto Mars. Moreover, the rise of the space development business may have already begun with the recent success of the Ansari X-Prize, a privately funded trans-atmospheric vehicle competition. However, the process of descending from space into an atmosphere must be handled with extreme care because any subtle failure could lead to destruction and death. For example, in 2003 the Columbia Space Shuttle broke apart in the atmosphere upon reentry because of a damaged reinforced carbon-carbon panel, caused by falling foam insulation from an external tank pylon during ascent to space. For this reason, it is very important to hone entry phase prediction capability. In pursuit of this goal, it is necessary to understand the physical phenomena that affect an entry vehicle during this phase of flight. This knowledge is gained from a combination of theory and experiment. Because of the hypervelocities experienced by entry vehicles there is significant expense and danger associated with flight and windtunnel testing. These factors are mitigated by computer simulation, generating data from theory, and by comparing with existing windtunnel and flight data. Computer simulation alone does not obviate the need for physical experimentation, but it can greatly reduce the amount of such experimentation.

There are two major descriptors of entry vehicles. Those that return to the atmosphere they came from are often called reentry vehicles. Those that travel to other planets and moons with atmospheres are referred to as entry vehicles. Since the analysis of this paper applies to both, the more general description of entry vehicles is employed. Entry vehicles generally slow down during atmospheric entry. Deceleration may be achieved by aerobraking, retropropulsion, a non-aerodynamic external force, or any combination of these. In this paper, only aerobraking mechanisms are considered. Most aerobraking entry vehicles have a blunt or bluff body structural configuration. There are three major kinds of blunt bodies: winged bodies, lifting bodies and ballistic capsules. The Space Shuttle Orbiter exemplifies the winged body. The X-38, a prototype design for an emergency crew-return vehicle from the International Space Station, is an example of a lifting body. It has stubby wing-like protrusions in the tail mainly for stability and control. Finally, for the ballistic capsule, there are a few well-known examples. In 1961, the first man in space was returned by the Soviet Vostok. Other ballistic capsules of 1960's vintage included the Mercury, Gemini and Apollo. The present expedition crews to the ISS employ the Russian Soyuz. The Crew Exploration Vehicle that will replace the space shuttle is of this module-capsule type of configuration. There are also various unmanned ballistic capsule entry vehicles. These include the vehicles that successfully entered the atmosphere of Mars and those that have returned to the Earth from science missions.

The analysis of this paper targets aerobraking entry vehicles with simple geometry because of available data and relevance to the present aerospace technology. In the mid to late 1960's, hypersonic wind-tunnel tests of small scale models were performed. ${ }^{1-4}$ The tests were performed at the Arnold Engineering Development Center (AEDC) in the von Karman Gas Dynamics Facility (VKF) and involved a low density, hypersonic, continuous-flow, arc heated, and ejector-pumped windtunnel called VKF Tunnel L. For this report, the aerodynamics of three small scale models in these windtunnel tests is examined. First, descriptions of the computational approaches are given. Second, the gas flow conditions and computational grids are presented. Current computational mesh-generation techniques are employed to define computational domains. Third, the numerical results are presented. In the windtunnel study that examined various vehicle shapes, ${ }^{1}$ data was compared with modified Newtonian and free molecular flow analysis. In this study, these flow analyses are reproduced and DSMC analysis is added. The purpose is to embark on the process of improving DSMC physical modeling procedures for transitional to rarefied hypersonic gasdynamic environments of entry vehicles. Serendipitously, this study brings to attention potential errors in some of the hypersonic 
windtunnel test data from the 1960s. Finally, conclusions based on the computational results and the existing windtunnel data are presented.

\section{Modeling Approaches}

\section{A. Flow Regime and Relevance}

Numerical models can be selected for gas dynamic simulation based on free-stream speed and Knudsen number. The windtunnel tests, which simulated aerobraking entry vehicles at the initial phase of atmospheric entry, involved gas flow traveling at thousands of meters-per-second with respect to the vehicle. A Newtonian model for solids immersed in a gas flow can be used to analyze these hypervelocity gases. This model can be used with a flat panel surface approximation to provide a simple numerical calculation to estimate the vehicle aerodynamic properties. Further details on this approach are described below. The Knudsen number of each windtunnel test was on the order of $10^{-1}$. This Knudsen number is in the upper range of the transitional flow regime, the flow regime that lies between the rarefied flow regime $\left(K n>10^{-1}\right)$ and the continuum flow regime $\left(K n<10^{-4}\right)$. When the local flow is in the rarefied regime or the upper range of the transitional regime, it is best modeled by a kinetic approach. When the flow is highly rarefied $(K n>>1)$, it can be described accurately with free molecule or collisionless flow kinetic theory. The free molecular flow model can only provide limiting values on the vehicle aerodynamics when applied to transitional flow. In the transitional regime itself, the flow is accurately and efficiently described by the direct simulation Monte Carlo (DSMC) $)^{5}$ method.

Primitive modeling approaches often provide a stepping stone to the more sophisticated numerical approaches. From a conceptual design perspective, primitive approaches provide initial estimates of vehicle performance from where a second design iteration may begin using more sophisticated and expensive methods. From an analysis perspective, the less sophisticated approaches provide simple functions to help develop auxiliary functions, such as aerodynamic coefficient integration procedures, that will eventually be used with more complicated procedures. From a theoretical perspective, the less sophisticated theory often helps identify phenomena and their effects. The conceptual design process, computer program development, and theoretical analysis provide three reasons for using primitive modeling approaches.

In this paper, modified Newtonian theory and free molecular flow analysis help develop procedures to compute aerodynamic properties from DSMC analysis of the windtunnel tests. The aerodynamic post-processing procedures are developed to help analyze future modifications or additions of physical models within the DSMC approach.

\section{B. Modified Newtonian Flow}

Over three centuries ago, Isaac Newton made propositions to determine the pressure of simple shapes, such as spheres, submerged in a steady uniform stream of a "rare medium". ${ }^{6-8}$ Incidentally, the involved mechanics provide a rough estimation of vehicle aerodynamic properties in hypersonic flow. In Newton's model, the flow is comprised of rectilinear streams of particles. The particles are assumed to lose all their normal momentum upon striking the vehicle surface and then move tangential to the surface. Application of Newtonian dynamics gives an expression for the surface pressure distribution, depending only on the local surface inclination angle relative to the free-stream. In dimensionless form, the expression is known as the Newtonian sine-squared law. A modification to the model, making it semi-empirical, incorporates the value of the maximum pressure coefficient,

$$
\mathrm{C}_{\mathrm{p}}=\mathrm{C}_{\mathrm{p}_{\max }} \sin ^{2} \theta_{\mathrm{b}}
$$

where

$$
-\sin \theta_{b}=\frac{\mathbf{V}_{\infty}}{\left|\mathbf{V}_{\infty}\right|} \cdot \mathbf{n}
$$

When $180^{\circ}<\theta_{\mathrm{b}}<360^{\circ}$, the surface pressure is set to the free-stream pressure, viz. $C_{p}=0$. The associated geometry is illustrated in Fig. 1. In this figure, the vectors $\mathbf{V}_{\text {particle, i, }}, \mathbf{n}$, and $\mathbf{V}_{\text {particle, }}$ lie in the same plane. This flow model, with a good estimate of $\mathrm{C}_{\mathrm{p}_{\max }}$, can provide an inexpensive estimate of hypersonic transitional flow aerodynamics for configurations where pressure dominates over shear stress. 


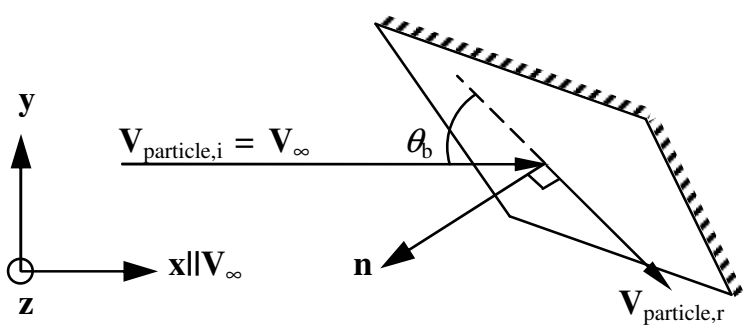

Figure 1. Surface Element of Newtonian Flow

\section{Free Molecular Flow}

When the Knudsen number exceeds 10, collisions between particles become so few that the gas can be considered collisionless. The motion of this gas is called a free molecule or a free molecular flow. Properties of rarefied gases experienced in a low Earth orbit above $150 \mathrm{~km}$ for a vehicle with a 1 meter characteristic length can be accurately computed by using free molecular flow analysis. ${ }^{9}$ The properties of interest in entry vehicle analysis are those that affect the vehicle's performance. These include the pressure, shear stress and heat flux. Note, free molecular flow analysis is not to be used as an initial estimate of transitional flow aerodynamics in the conceptual design process; rather, it is valid for predicting aerodynamics of satellites at $150 \mathrm{~km}$ altitude and above. The reason for its inclusion in this study is to provide physical insight and program development. In a free molecular flow, the mean distance between intermolecular collisions is much greater than the characteristic size of the vehicle. The vehicle is assumed to be immersed in an infinite domain of a collisionless gas having a Maxwellian velocity distribution. Because molecules approaching the vehicle surface do not collide with reflected molecules, the freestream molecules receive no warning about the approaching vehicle and collide with its surface with the free-stream particle or molecular velocity. Furthermore, the particles reflect from the vehicle surface in a fully or partially diffuse manner with a Maxwellian velocity distribution. The degree of diffuse reflection is governed by tangential $\left(\sigma_{t}\right)$ and normal $\left(\sigma_{n}\right)$ momentum accommodation coefficients. Under these conditions, expressions for the surface properties can be derived. Consider a flat surface element with a local coordinate system defined by the orthonormal set of vectors $(\mathbf{u}, \mathbf{v}, \mathbf{w})$, where $\mathbf{u}$ is parallel to one side of the element and the origin is at a corner, Fig. 2. Vectors $\mathbf{u}$ and $\mathbf{v}$ lie in the same plane as the polygonal (triangular, rectangular, etc.) surface element.

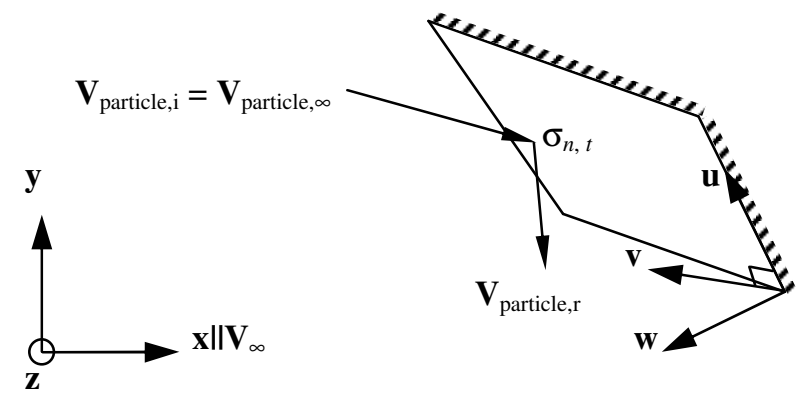

Figure 2. Surface Element of Free Molecular Flow

It can be shown that the corresponding surface pressure and shear stress are: ${ }^{10}$

$$
\begin{aligned}
p=q_{\infty}\left(\frac{\sigma_{n}}{2} \sqrt{\frac{T_{w}}{T_{i}}}\left\{\frac{e^{-w_{x}^{2} s^{2}}}{s^{2}}-\frac{w_{x} \sqrt{\pi}}{s}\left[1+\operatorname{erf}\left(-w_{x} s\right)\right]\right\}\right. \\
\left.-w_{x}\left(2-\sigma_{n}\right)\left\{\frac{e^{-w_{x}^{2} s^{2}}}{s \sqrt{\pi}}-w_{x}\left[1+\operatorname{erf}\left(-w_{x} s\right)\right]\right\}+\frac{2-\sigma_{n}}{2 s^{2}}\left[1+\operatorname{erf}\left(-w_{x} s\right)\right]\right)
\end{aligned}
$$




$$
\tau_{u}=q_{\infty} \sigma_{t} u_{x}\left\{\frac{e^{-w_{x}^{2} s^{2}}}{s \sqrt{\pi}}-w_{x}\left[1+\operatorname{erf}\left(-w_{x} s\right)\right]\right\}
$$

and

$$
\tau_{v}=q_{\infty} \sigma_{t} v_{x}\left\{\frac{e^{-w_{x}^{2} s^{2}}}{s \sqrt{\pi}}-w_{x}\left[1+\operatorname{erf}\left(-w_{x} s\right)\right]\right\}
$$

where $q_{\infty}$ is the free-stream dynamic pressure and $s$ is the molecular speed ratio:

$$
\begin{gathered}
q_{\infty}=\frac{1}{2} \rho_{\infty} V_{\infty}^{2} \\
s=\frac{V}{V_{\mathrm{mp}}^{\prime}}=\sqrt{\frac{\gamma}{2}} M a
\end{gathered}
$$

\section{Direct Simulation Monte Carlo Method}

Central to this analysis of transitional hypersonic flows is the DSMC method. It is a physically based probabilistic numerical simulation method. It is not a direct numerical solution to the classical Boltzmann equation, but uses the same underlying physics. Unlike the deterministic molecular dynamics method, it probabilistically selects collision processes for analysis. The procedures used to simulate these collision processes are based on kinetic theory. Hence, the method is limited to dilute gases with thermochemical behavior. This more than encompasses the significant physical behavior of the flow about an entry vehicle model during a windtunnel test.

This study employs the general, object-oriented, cell-based, parallelized implementation of the DSMC method called MONACO. ${ }^{11}$ It uses the Variable Soft Sphere (VSS) collision model, ${ }^{12}$ and variable vibrational ${ }^{13}$ and rotationa $1^{14}$ energy exchange probability models. When chemical reactions are relevant, the Total Collision Energy (TCE) $\operatorname{model}^{5}$ regulates the reactions. The code can handle structured and unstructured meshes in two or three spatial dimensions. It employs a thermal energy accommodation coefficient $a_{\mathrm{c}}$ to regulate interactions between particles and a wall surface, as illustrated in Fig. 3. This coefficient indicates the probability that a particle's translational energy will change according to diffuse reflection and that its rotational and vibrational energy will change randomly according to the wall temperature; otherwise, specular reflection is assumed and the internal energies are unchanged. When the mean translational energy of gas molecules relative to the surface is several electron volts, the assumption of full thermal accommodation is not generally valid. ${ }^{5}$ An example of this condition is the Apollo 6, $110 \mathrm{~km}$ entry-trajectory point, ${ }^{15}$ where the mean molecular translational energy is about $14 \mathrm{eV}$. In this study, the mean translational energy of the gas is on the order of $1 \mathrm{eV}$, and for a set of simulations, $a_{\mathrm{c}}$ is reduced slightly from unity in order to examine the corresponding sensitivity of the vehicle aerodynamics.

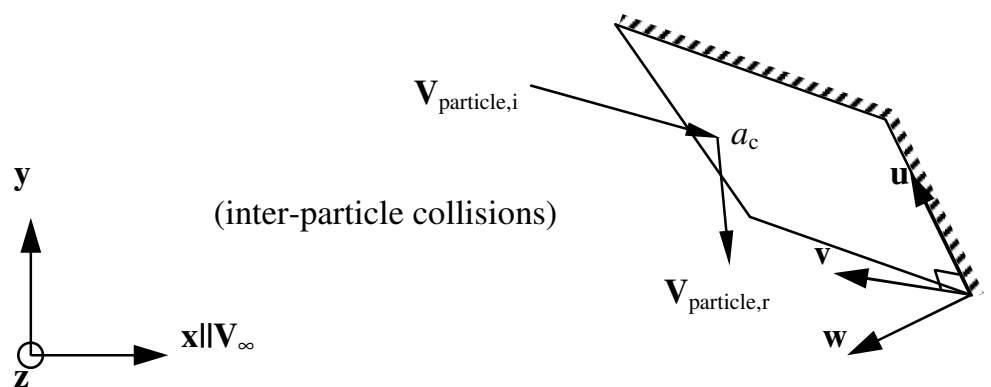

Figure 3. Surface Element used in MONACO 


\section{E. Post Processing}

All of the above approaches are used to compute aerodynamic coefficients of selected windtunnel test models. They employ the same overall procedure. For example, for the aerodynamic force coefficients: (1) compute forces due to pressure and shear stress at a wall element, (2) resolve them along a desired direction, (3) sum over all elements representing the vehicle surface to determine lift, drag, normal force or axial force, and (4) nondimensionalize. The same steps are involved to resolve the vehicle pitching moment, except with a cross product relative to a specified reference point taken at each element between steps (1) and (2).

\section{Windtunnel Simulation Descriptions}

\section{A. Flow Conditions and Vehicle Models}

Flow conditions and geometry for the blunted-cone windtunnel test ${ }^{1}$ are shown in Table 1 and Fig. 4, respectively. The pitching moment for the $9^{\circ}$ blunted-cone is taken with respect to point $\mathrm{O}$. More details of the cone's geometry are found in Ref. 1. The corresponding information for the Apollo Command Module (ACM) windtunnel test ${ }^{3,4}$ is shown in Table 2 and Fig. 5. Figure 5 shows the location of the center-of-gravity with respect to the windward heat-shield apex and defines the angle-of-attack with respect to the leeward vehicle axis of symmetry. The pitching moment for the ACM is taken relative to the center-of-gravity. Further details of the ACM geometry are found in Ref. 4.

Table 1. Conditions for the blunted-cone windtunnel test

\begin{tabular}{|l|l|}
\hline Property & Value \\
\hline Base diameter $d$ & $1.524 \times 10^{-2} \mathrm{~m}$ \\
Gas & $\mathrm{N}_{2}$ \\
$\lambda_{\infty}$ & $9.9 \times 10^{-4} \mathrm{~m}$ \\
$T_{\infty}$ & $143.5 \mathrm{~K}$ \\
$M a_{\infty}$ & 10.15 \\
$R e_{\mathrm{d}}$ & 233 \\
$T_{w}$ & $600 \mathrm{~K}$ \\
$K n$ & 0.065 \\
$n_{\infty}$ & $1.323 \times 10^{21} \mathrm{~m}^{-3}$ \\
$V_{\infty}$ & $2478 \mathrm{~m} / \mathrm{s}$ \\
\hline
\end{tabular}

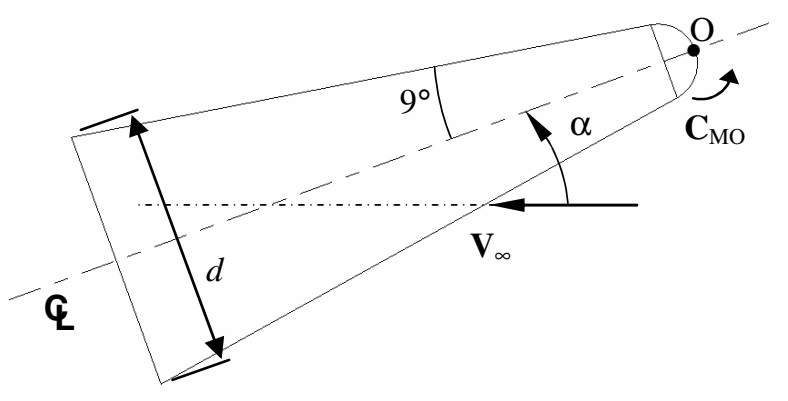

Figure 4. Blunted-cone Geometry

Table 2. Conditions for the ACM windtunnel test

\begin{tabular}{|l|l|}
\hline Property & Value \\
\hline Vehicle Diameter $d$ & $1.524 \times 10^{-2} \mathrm{~m}$ \\
Gas & $\mathrm{N}_{2}$ \\
$T_{\infty}$ & $142.2 \mathrm{~K}$ \\
$M a_{\infty}$ & 10.20 \\
$R e_{\mathrm{d}}$ & 230 \\
$T_{w}$ & $300 \mathrm{~K}$ \\
$K n$ & 0.067 \\
$n_{\infty}$ & $1.280 \times 10^{21} \mathrm{~m}^{-3}$ \\
$\lambda_{\infty}$ & $0.001 \mathrm{~m}$ \\
$V_{\infty}$ & $2479 \mathrm{~m} / \mathrm{s}$ \\
\hline
\end{tabular}

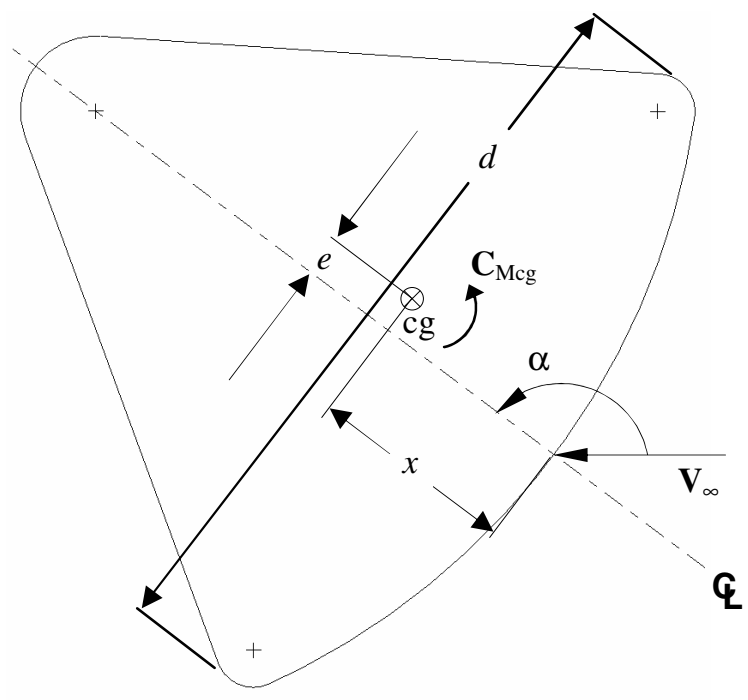

Figure 5. ACM Geometry 
A windtunnel test of the Gemini Command Module (GCM) at 180 degrees angle-of-attack is also simulated. The conditions for this windtunnel test are the same as those for the blunted-cone windtunnel test, except for the vehicle reference length, and consequently, vehicle Knudsen number. The reference length is the model diameter of $d=1.27$ $\mathrm{cm}$. The corresponding vehicle Knudsen number and Reynolds number are $K n=0.078$ and $R e_{\mathrm{d}}=194$, respectively. The reference length or diameter of the Gemini model is 17\% smaller than that of the Apollo model. Figure 6 shows the geometry. Further details of the GCM geometry are found in Refs. 1 and 16.

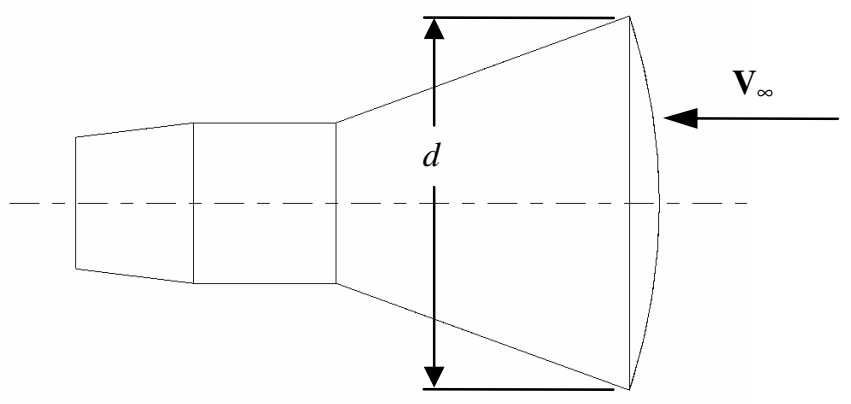

Figure 6. GCM Geometry

\section{B. Computational Meshes}

Three-dimensional computational domain boundaries are generated for the blunted-cone and the ACM. The computational domain boundary surfaces are generated with ProE. ${ }^{17}$ They are then exported to HyperMesh, ${ }^{18}$ where the interior unstructured tetrahedral mesh is created. Figures 7 (a) and (b) are images of the domain boundary surface meshes. Three-dimensional simulations are made for the blunted-cone at 0, 10, 20 and 25 degrees angle-ofattack, and for the ACM at 180, 170, 160, 150 and 140 degrees angle-of-attack.

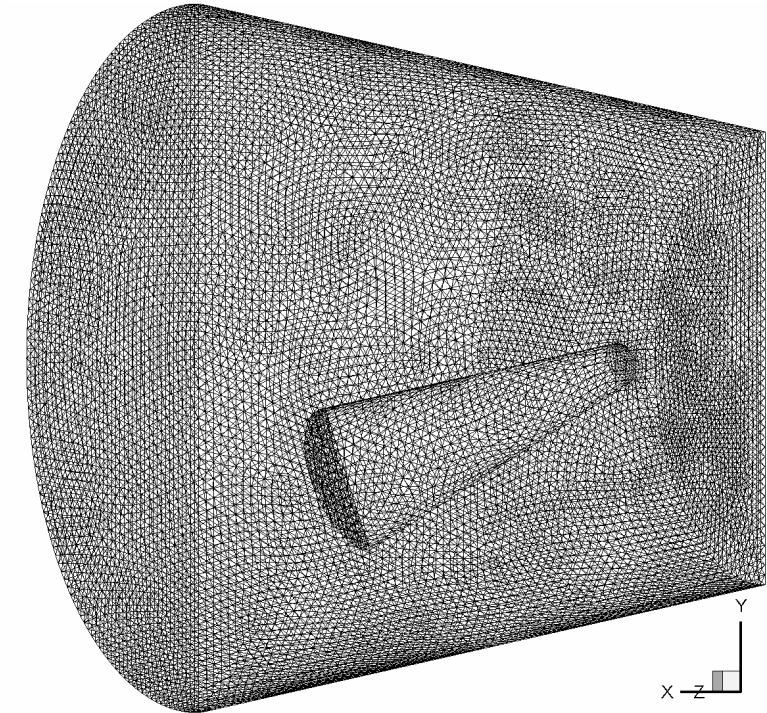

(a) Entire Blunted-cone Simulation Boundary with Blunted-cone at a $20^{\circ}$ angle-of-attack

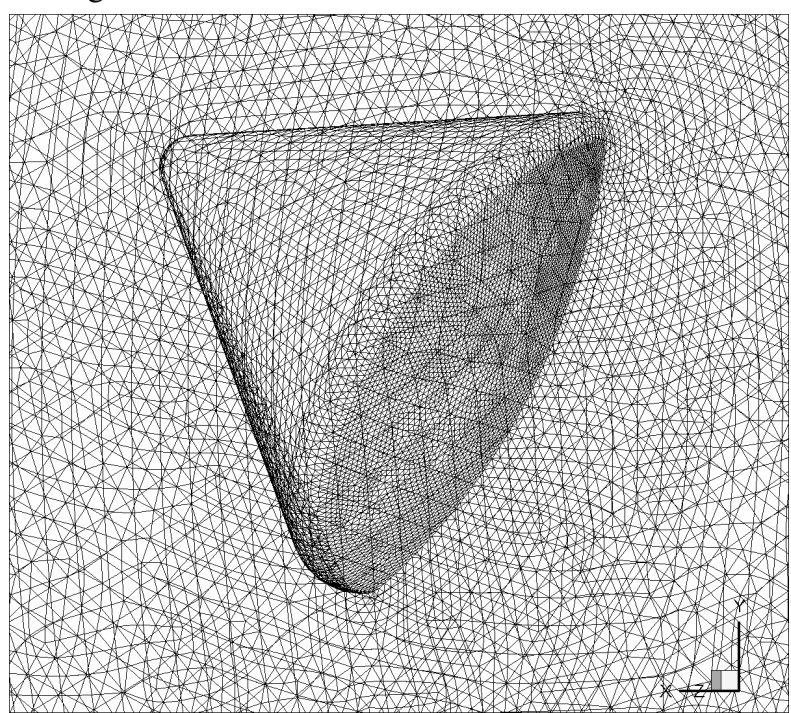

(b) Simulation Boundary near the ACM at a $150^{\circ}$ angle-of-attack

\section{Figure 7. Images of Domain Boundary Surface Meshes for the Three-Dimensional Simulations}

Two-dimensional computational meshes are generated for the Apollo and Gemini models at 180 degrees angleof-attack, where they have axisymmetry. Domain boundaries are also generated with ProE and exported to HyperMesh, where the interior triangular or quadrilateral cell mesh is created. Figures 8 (a) and (b) are images of these meshes. Quadrilateral cells are employed for these studies. 


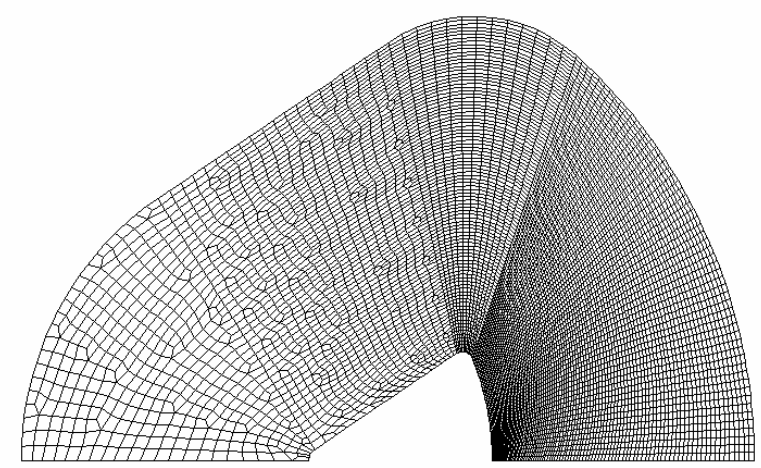

(a) Apollo Command Module

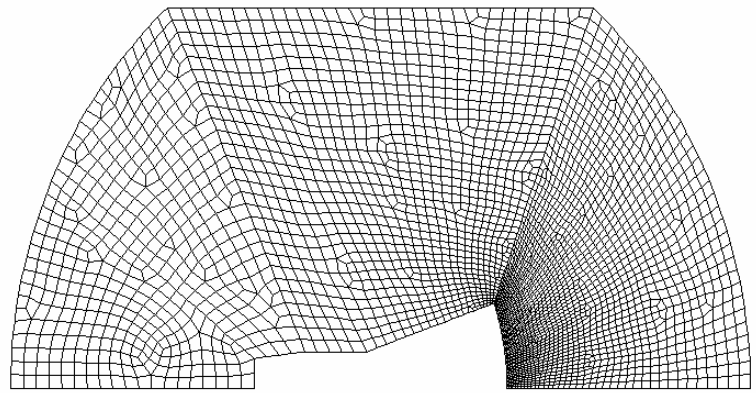

(b) Gemini Command Module

Figure 8. Images of the Axisymmetric Simulation Meshes with Quadrilateral Cells

\section{Simulation Numerical Parameters}

Each of the gas flow models described above is applied to compute aerodynamic coefficients for the bluntedcone and the ACM windtunnel models, under the windtunnel test conditions. For the modified Newtonian and free molecular flow calculations, only the vehicle surface grid is necessary. The surface grids are extracted from the three-dimensional grids employed in the DSMC calculations. The blunted-cone and ACM surface grids have 1,915 and 8,957 cells, respectively. The larger number of cells for the ACM surface grid reflects the larger compression region ahead of the vehicle that requires smaller cells in that region of the three-dimensional DSMC calculations. These surface computations are performed on a single $3 \mathrm{GHz}$ class Intel processor. For each entry vehicle, the computations take less than 30 seconds to produce lift, drag and moment coefficients for seven angles-of-attack. The free molecular flow calculations assume diffuse reflection of particles from the vehicle surface $\left(\sigma_{n}=\sigma_{t}=1\right)$.

For the DSMC calculations, each angle-of-attack result requires a separate simulation. Furthermore, for the blunted-cone, two simulations are made at each angle-of-attack corresponding to different gas-surface thermal energy accommodation coefficients. These simulations employ three-dimensional unstructured grids with tetrahedral cells. A total of 8 blunted-cone and 5 ACM simulations are made. Each blunted-cone grid has roughly 560,000 cells and each ACM grid has about 740,000 cells. Again, the larger number of cells for the ACM reflects the larger compression region ahead of the vehicle due to the greater degree of bluntness of the ACM. The free-stream regions are sufficiently large to capture the diffuse shockwave structure within the vicinity of the entry vehicles. The peak flow temperature is not sufficiently large to activate chemical reaction procedures. Table 3 summarizes the numerical parameters of these simulations.

Table 3 Typical Numerical Parameters

\begin{tabular}{|l|rrrcc|}
\hline Simulation & \multicolumn{1}{|c}{ Cells } & Particles & Time Step (s) & Steps & Processors $^{* * *}$ \\
\hline $\begin{array}{l}\text { Axisymmetric } \\
\text { Apollo }\end{array}$ & 9,332 & $1,320,000$ & $1.5 \times 10^{-8}$ & 40,000 & 2 \\
Gemini & 3,348 & 818,000 & $1.5 \times 10^{-8}$ & 40,000 & 1 \\
& & & & & \\
3-Dimensional & & & & \\
$\begin{array}{l}\text { Blunted-cone } \\
\text { Apollo }\end{array}$ & 560,000 & $12,500,000$ & $2.0 \times 10^{-8}$ & 30,000 & 8 \\
\hline
\end{tabular}

*Quadrilateral Cells

${ }^{* * *}$ Tretrahedral Cells

*** $1.4-1.8 \mathrm{GHz}$ Opteron or Intel Itanium processors 


\section{Windtunnel Simulation Results}

\section{A. Validation of Aerodynamic Force Integration}

In order to validate the integration procedures used to compute the vehicle aerodynamic coefficients in threedimensional flow, the free molecular flow analysis is employed. It provides pressure and shear stress expressions for flat panel surface representations, equations (3) through (5), and it is amenable to exact integral equations ${ }^{10}$ for the aerodynamic coefficients of simple shapes. For the blunted-cone, the aerodynamic coefficients are found from:

$$
\begin{gathered}
q_{\infty} S C_{D}=\iint_{\substack{\text { cone } \\
\text { frustrum }}}\left(\frac{d F}{d A}\right)_{D} d A+\iint_{\substack{\text { spherical } \\
\text { nose }}}\left(\frac{d F}{d A}\right)_{D} d A \\
q_{\infty} S C_{L}=\iint_{\substack{\text { cone } \\
\text { frustrum }}}\left(\frac{d F}{d A}\right)_{L} d A+\iint_{\substack{\text { spherical } \\
\text { nose }}}\left(\frac{d F}{d A}\right)_{L} d A \\
q_{\infty} S d C_{M}=\iint_{\substack{\text { cone } \\
\text { frustrum }}}\left[\operatorname{arm}_{D}\left(\frac{d F}{d A}\right)_{D}+\operatorname{arm}_{L}\left(\frac{d F}{d A}\right)_{L}\right] d A+\iint_{\substack{\text { spherical } \\
\text { nose }}}\left[\operatorname{arm}_{D}\left(\frac{d F}{d A}\right)_{D}+\operatorname{arm}_{L}\left(\frac{d F}{d A}\right)_{L}\right] d A
\end{gathered}
$$

where ( $d F / d A)_{\mathrm{D}, \mathrm{L}}$ represents the components of pressure and shear stress along the drag or lift direction, and sphere or cone area differentials are employed. The functions $\operatorname{arm}_{D, L}$ represent the respective vertical and horizontal moment arms for the pitching moment. The resultant pressure and shear force on a surface element are obtained from

$$
\begin{aligned}
\frac{1}{q_{\infty}} \frac{d F}{d A}=\left[\sigma_{t}(\varepsilon k+\eta t)+\left(2-\sigma_{n}\right)\right]\left\{\zeta s^{2}[1+\operatorname{erf}(\zeta s)]+\frac{s}{\sqrt{\pi}} e^{-\zeta^{2} s^{2}}\right\} \\
+\frac{\left(2-\sigma_{n}\right) l}{2}[1+\operatorname{erf}(\zeta s)]+\frac{\sigma_{n} l}{2} \sqrt{\frac{T_{w}}{T_{i}}}\left\{\zeta s \sqrt{\pi}\left[1+\operatorname{erf}(\zeta s)+e^{-\zeta^{2} s^{2}}\right]\right\}
\end{aligned}
$$

The results of equations (3) through (5) summed over a flat panel representation of the blunted-cone are compared with the results of equations (8) through (10). The precise agreement between the integration procedures is demonstrated in Fig. 9. The variations of $L / D$ and $\mathrm{C}_{\mathrm{MO}}$ as functions of angle-of-attack are governed by the vehicle shape. They are odd functions about $180^{\circ}$ because of the axisymmetry. The $L / D$ variation is primarily governed by the lift variation. The angle of maximum $L / D$, which is $20^{\circ}$, is also the angle of maximum lift. The $L / D$ becomes negative between $75^{\circ}$ and $80^{\circ}$ because that is where the exposed $9^{\circ}$ half angle blunted-cone begins exhibiting negative lift. The $L / D$ increases between $110^{\circ}$ and $170^{\circ}$ because that is where the cone base becomes exposed to the free-stream and produces increasing lift. The sinusoidal behavior of the pitching moment variation can be attributed to the approximate flat plate shape of the slender blunted-cone. The plateau regions of the $\mathrm{C}_{\mathrm{MO}}$ curve occur when the cone base becomes exposed to the free-stream; and thus, are due to counteracting moment components from the cone base. 


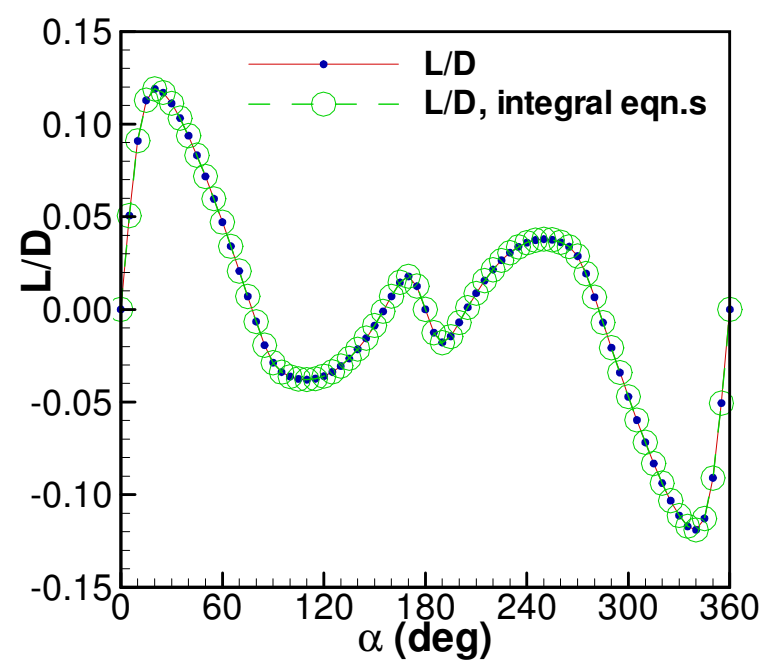

(a) Lift-to-Drag Ratio

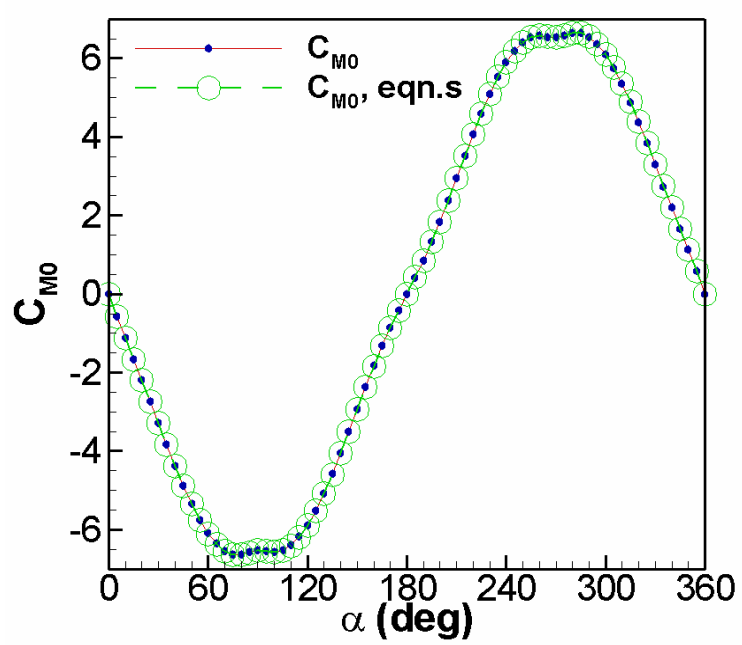

(b) Pitching Moment

Figure 9. Validation of numerical integration procedures against exact integral equations using free molecular flow analysis for the blunted-cone at $M a_{\infty}=9.56$

\section{B. Blunted-cone Simulations}

The DSMC method is suitable for computing pressure and shear stress distributions over the vehicle surface for the transitional to rarefied flow conditions of the windtunnel tests described above. These distributions are integrated, with the same procedures used to integrate the free molecular flow pressure and shear stress distributions, to obtain the overall vehicle aerodynamic coefficients. Figures 10 (a) and (b) illustrate the general character of the flow field for the blunted-cone. The free-stream region is sufficiently large to capture the diffuse shock near the vehicle and the peak flow temperature is not sufficient to justify activation of nitrogen dissociation procedures.

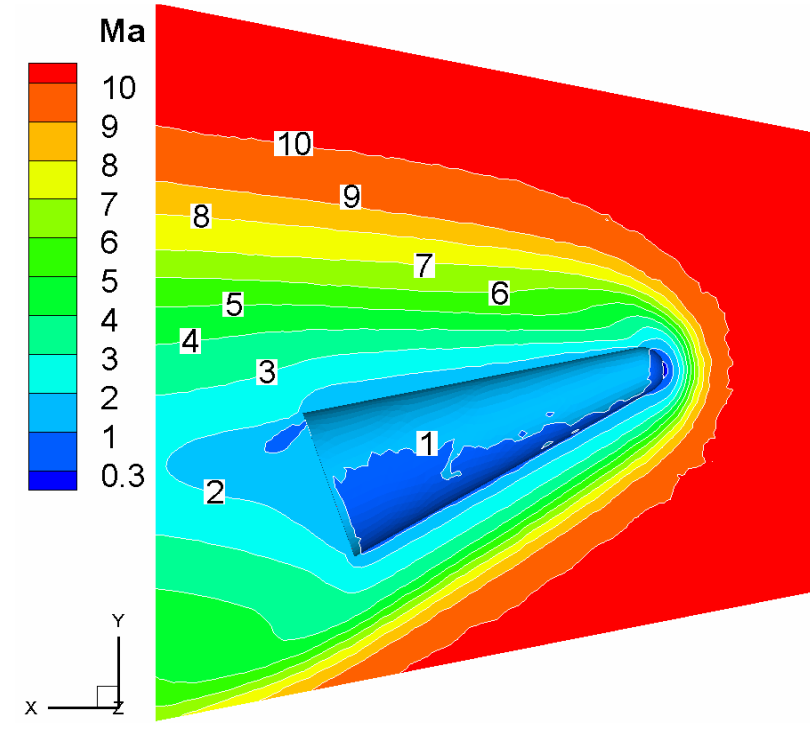

(a) Blunted-cone Mach Number

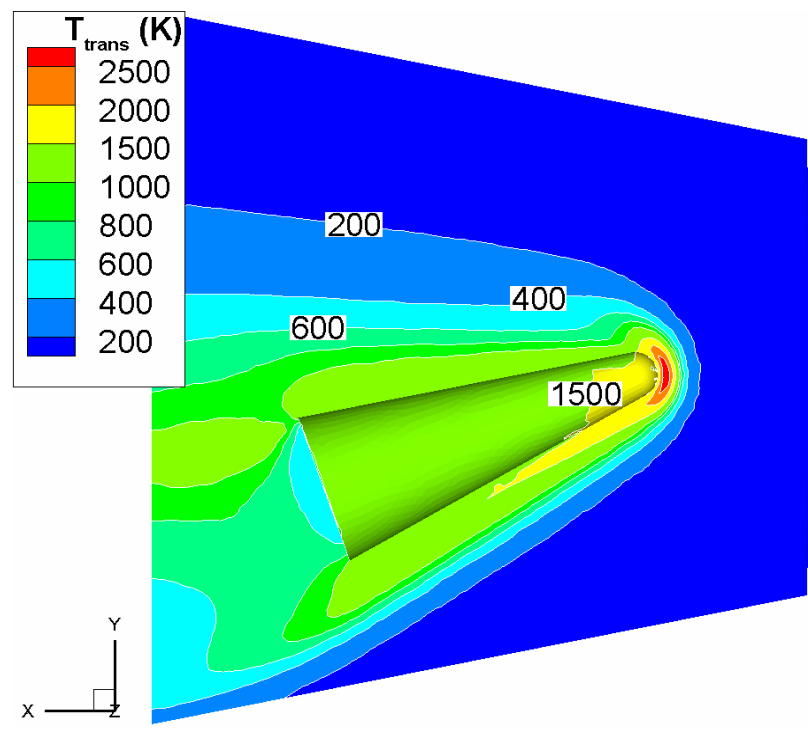

(b) Blunted-cone Translational Temperature

Figure 10. Contour Plots of the Three-Dimensional Simulations with the blunted-cone at a $20^{\circ}$ angle-of-attack 
The aerodynamic results of the DSMC simulations for the blunted-cone, with two values of accommodation coefficient, are compared with the simpler flow models and the available windtunnel data. Figure 11 (a) compares the drag coefficient from the computations and the windtunnel tests at various angles-of-attack. In these figures, the DSMC calculations generally yield lift and drag coefficients that lie somewhere between the values given by the modified Newtonian and free molecular flow results. A major reason the modified Newtonian model predicts lower drag is that it neglects shear stress. The free molecular flow model predicts higher drag because it neglects intermolecular collisions. The modified Newtonian analysis for the blunted-cone employed $\mathrm{C}_{\mathrm{p}_{\max }}=1.83$, from Ref.

1. The variation of $\mathrm{C}_{\mathrm{D}}$ with $\alpha$ given by the DSMC method agrees well with that of the windtunnel data. The DSMC and windtunnel data are 6.9, 8.7, 7.8 and 7.7\% different for $0,10,20$ and $25^{\circ}$, angle-of-attack, respectively. At $0^{\circ}$ angle-of-attack, a reduction in the thermal accommodation coefficient by $15 \%$ reduces the drag coefficient by $6 \%$, from 0.82 to 0.77 . Figure 11 (a) shows that the effect is uniform for all the angles-of-attack considered. This moves the DSMC results slightly further from the windtunnel drag data because of reduced backscatter. Figure 11 (b) illustrates the comparisons for the lift coefficient. The DSMC lift trend also agrees well with the windtunnel data. The difference between the DSMC and windtunnel data is about $10 \%$ at 10,20 and $25^{\circ}$ angle-of-attack. The reduction in thermal accommodation coefficient does not significantly affect the lift coefficient.

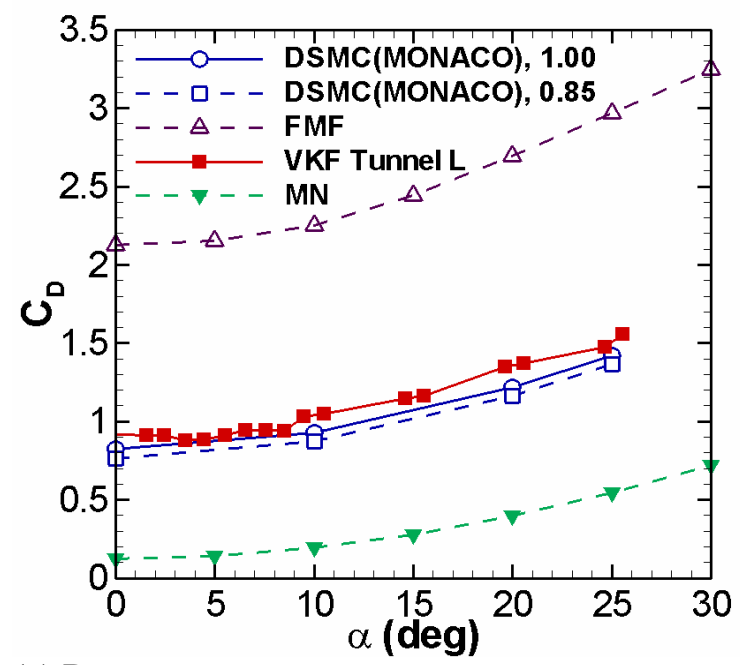

(a) Drag

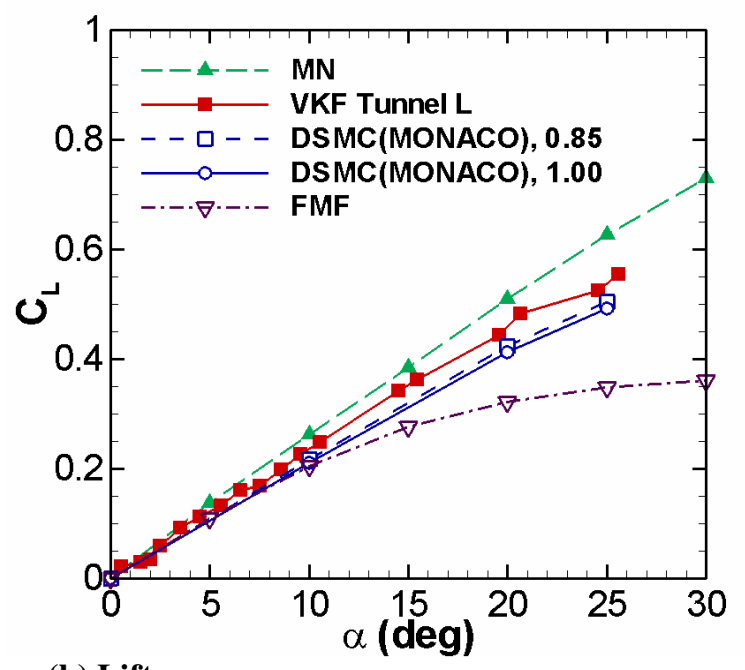

(b) Lift

Figure 11. Variation of Blunted-cone Drag and Lift with Angle-of-Attack MN = Modified Newtonian DSMC (code), accommodation coefficient

FMF = Free Molecular Flow VKF Tunnel $L=A E D C$ windtunnel

Figure 12 (a) illustrates the comparisons for the lift-to-drag ratio. Again, the reduction in thermal accommodation coefficient does not incur any significant effect. There is no significant difference between the DSMC and windtunnel data. Figure 12 (b) illustrates the comparisons for the pitching moment. Once again, the DSMC results lie between the simpler model results, the reduction in thermal accommodation coefficient does not incur any significant effect, and the DSMC and windtunnel data demonstrate good agreement. Percentage differences between the DSMC and windtunnel pitching moment are 11,5 , and $2 \%$ for $\alpha=10,20$ and $25^{\circ}$, respectively. 


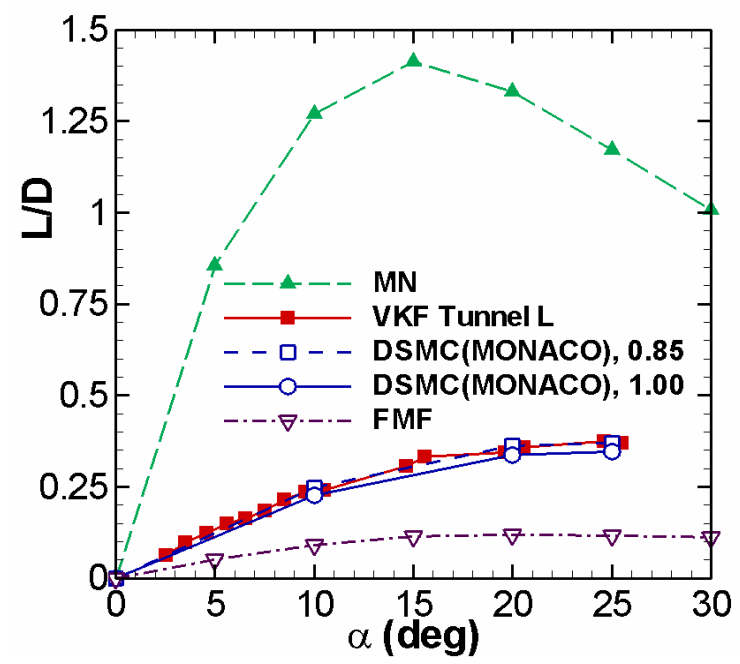

(a) Lift-to-Drag Ratio

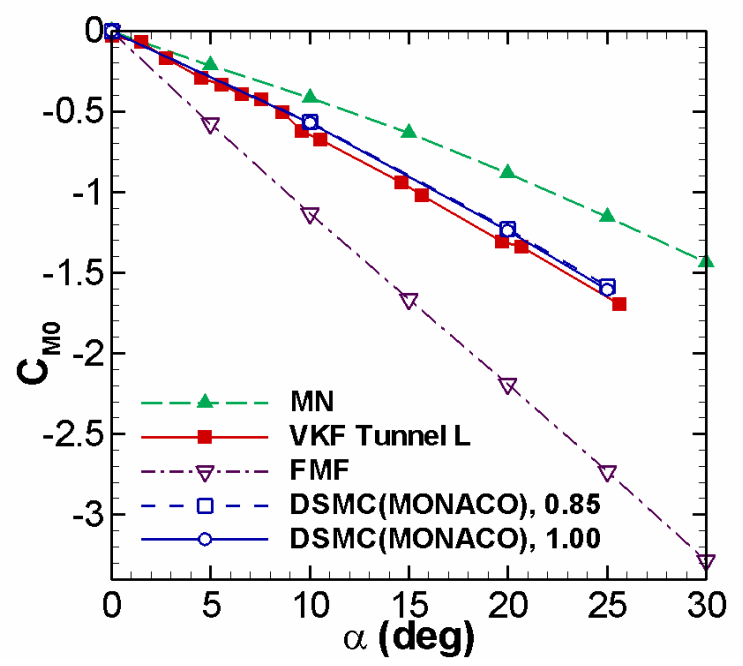

(b) Pitching Moment

Figure 12. Variation of Blunted-cone Lift-to-Drag Ratio and Pitching Moment with Angle-of-Attack MN = Modified Newtonian DSMC (code), accommodation coefficient

FMF = Free Molecular Flow

VKF Tunnel $\mathrm{L}=\mathrm{AEDC}$ windtunnel

\section{Apollo Command Module Simulations}

Similar simulations and comparisons are also made for the ACM windtunnel test. In these simulations, full thermal accommodation with diffuse reflection is employed. Figures 13 (a) and (b) illustrate the general character of the flow field for the ACM. The free-stream region is sufficiently large to capture the diffuse shock near the vehicle and the peak flow temperature is again not sufficiently large to justify activating chemical reaction procedures.
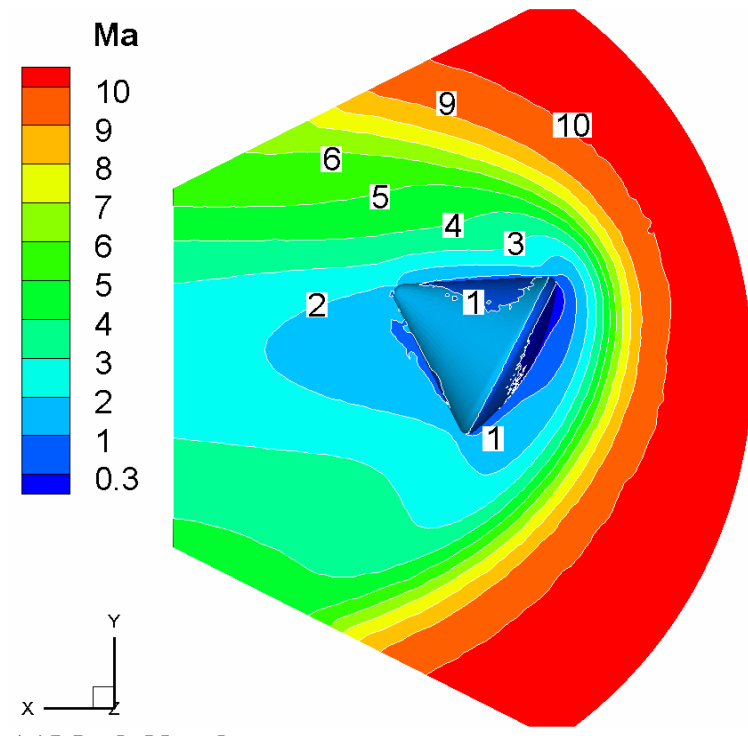

(a)Mach Number
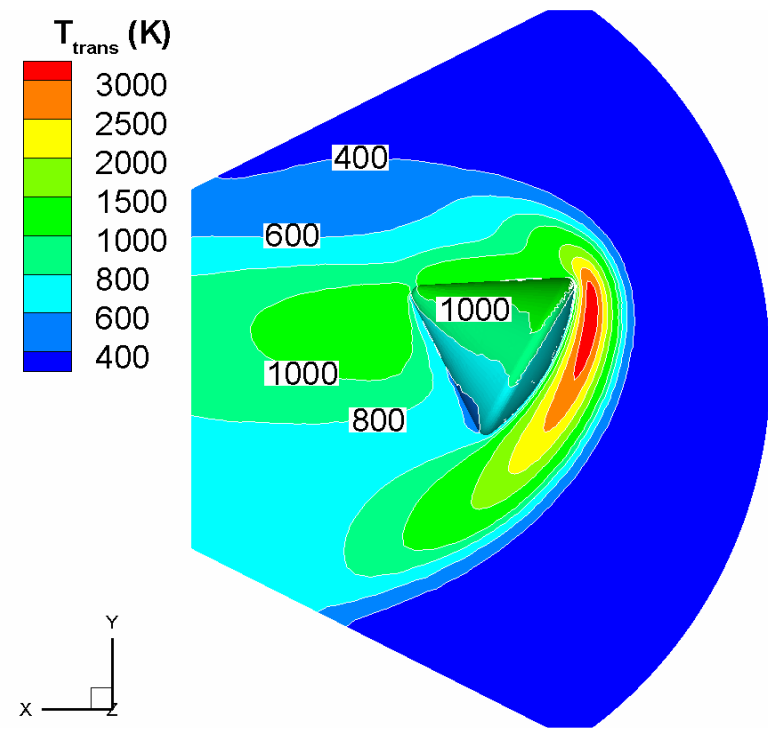

(b)

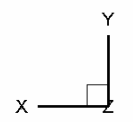

ACM Translational Temperature

Figure 13. Contour Plots of the Three-Dimensional Simulations with the $\mathrm{ACM}$ at a $150^{\circ}$ angle-of-attack

Figures 14 (a) through (d) compare $\mathrm{C}_{\mathrm{L}}, \mathrm{C}_{\mathrm{D}}, L / D$ and $\mathrm{C}_{\mathrm{Mcg}}$ at various angles-of-attack for the ACM between the windtunnel and computer data. In these figures, the DSMC calculations generally yield lift and drag coefficients that 
lie somewhere between the values given by the modified Newtonian and free molecular flow results. The modified Newtonian analysis involved $\mathrm{C}_{\mathrm{p}_{\max }}=1.89$ from the DSMC analysis. For the ACM, a second set of DSMC results, provided by Dr. James Moss, ${ }^{19}$ is also plotted. They provide confidence in the correctness of the MONACO procedures. The DSMC and windtunnel data do not agree well. At $180^{\circ}$, the DSMC and windtunnel drag coefficients are $12.6 \%$ different, almost twice the percentage difference than for the blunted-cone. The windtunnel drag decreases more rapidly with angle-of-attack than the DSMC drag. The windtunnel lift is substantially greater than any computational model. The windtunnel $\mathrm{C}_{\mathrm{D}}, \mathrm{C}_{\mathrm{L}}$ and $L / D$ were extracted from separate plots from Ref. 3. Taking the ratio of the DSMC lift and drag coefficients cancels out most of the disagreement between the DSMC results and the windtunnel data. This demonstrates a reason to plot the lift and drag coefficients separately when comparing aerodynamic simulation results with existing data. There is closer agreement between the DSMC and windtunnel pitching moment data than either the drag or lift. However, the agreement is still worse than for the blunted-cone.

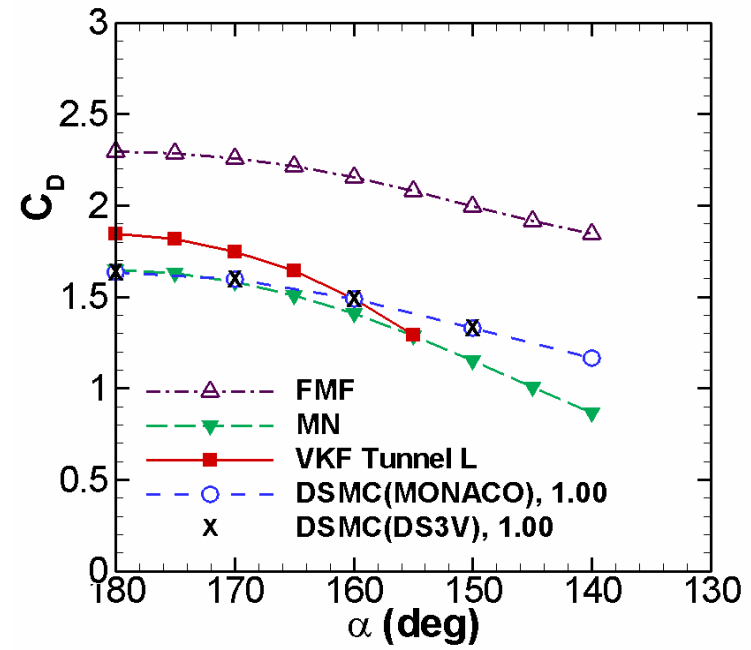

(a) Drag

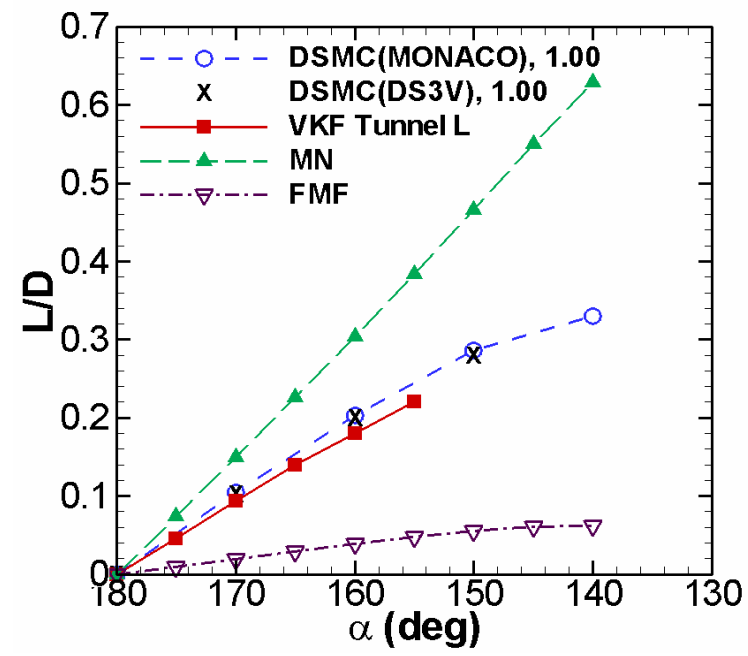

(c) Lift-to-Drag Ratio

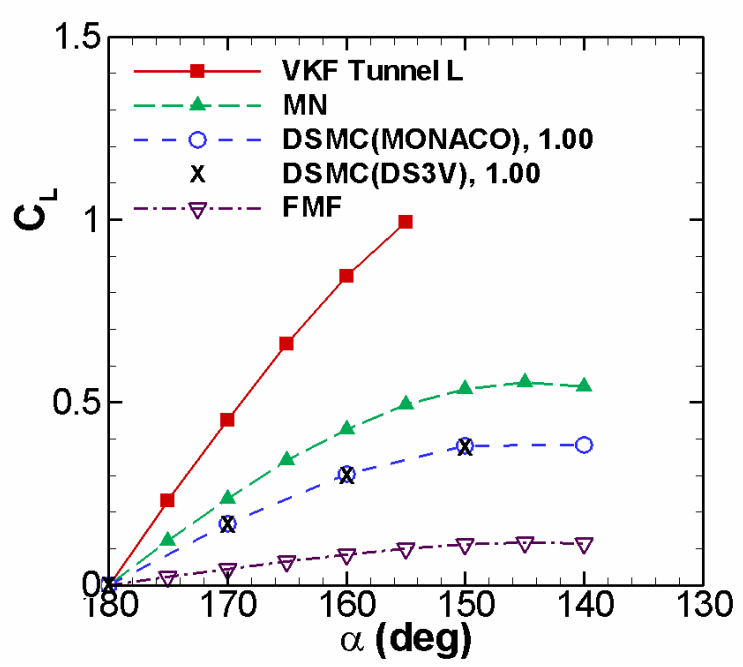

(b) Lift

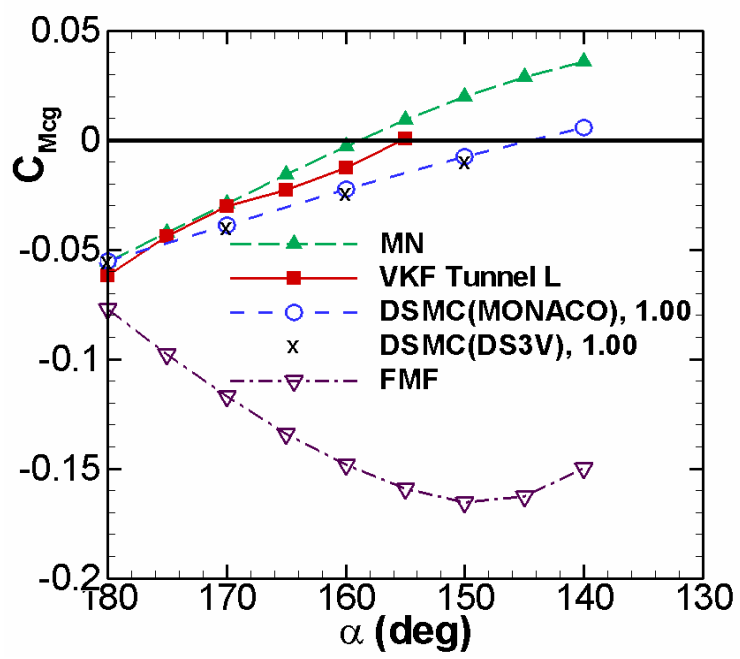

(d) Pitching Moment

Figure 14. Variation of ACM Drag and Lift with Angle-of-Attack

MN = Modified Newtonian

DSMC (code), accommodation coefficient
FMF = Free Molecular Flow

VKF Tunnel $L=$ AEDC windtunnel 


\section{Apollo and Gemini Axisymmetric Simulations}

When the axisymmetric windtunnel models are at a $180^{\circ}$ angle-of-attack, the axisymmetry allows one to simulate the flow with a two-dimensional grid that contains the vehicle profile. In general, for axisymmetric vehicles, these simulations are an inexpensive way of determining the general character of the flow-field, including required domain size and chemical activity, from where three-dimensional simulations may begin. They are also an inexpensive way of performing parametric analysis. In this study axisymmetric simulations are performed to determine whether the windtunnel data was possibly reported inaccurately by examining the sensitivity of the computed drag coefficient to changes in the reported conditions. This is performed by running simulations with the free-stream Mach number varied by $\pm 20 \%$ and with the reservoir temperature varied by $\pm 20 \%$. The corresponding free-stream temperature is computed from the Mach number and reservoir temperature, assuming isentropic flow. Then the free-stream speed of sound and bulk flow speed are determined. Table 4 provides the corresponding results. A $20 \%$ change in the free-stream Mach number corresponds to a roughly $30 \%$ or $40 \%$ change in the magnitude of the free-stream number density, but results in a less than $2 \%$ change in drag coefficient. The same variation in reservoir temperature gives smaller changes in free-stream conditions and insignificant changes in drag coefficient. Therefore, the reported conditions were reported accurately enough and the disagreement between the computer simulations and the windtunnel data for the drag coefficient must be due to some other problem. Possible explanations include flow non-uniformity, nozzle boundary layer effects and nonequilibrium free-stream conditions.

Table 4 Sensitivity of Drag Coefficient of ACM due to changes in Reported Conditions

\begin{tabular}{|c|c|c|c|c|c|c|}
\hline Baseline Conditions & $\begin{array}{r}M a_{\infty} \\
10.2\end{array}$ & 3100 & $\begin{array}{r}V_{\infty}(\mathrm{m} / \mathrm{s}) \\
2479\end{array}$ & $\begin{array}{c}n_{\infty} \\
\left(10^{21} / \mathrm{m}^{3}\right) \\
1.279\end{array}$ & $\begin{array}{l}C_{D} \\
1.645\end{array}$ & \\
\hline Variation & $V_{\infty}(\mathrm{m} / \mathrm{s})$ & $\Delta V_{\infty}$ & $\begin{array}{c}n_{\infty} \\
\left(10^{21} / \mathrm{m}^{3}\right)\end{array}$ & $\Delta n_{\infty}$ & $C_{D}$ & $\Delta \mathrm{C}_{\mathrm{D}}$ \\
\hline$M a_{\infty}$ decreased by $20 \%$ & 2448 & $-1.3 \%$ & 1.815 & $42 \%$ & 1.620 & $-2 \%$ \\
\hline$M a_{\infty}$ increased by $20 \%$ & 2497 & $0.7 \%$ & 0.9344 & $-27 \%$ & 1.679 & $2 \%$ \\
\hline$T_{0}$ decreased by $20 \%$ & 2217 & $-11 \%$ & 1.180 & $-8 \%$ & 1.646 & $0.1 \%$ \\
\hline$T_{0}$ increased by $20 \%$ & 2716 & $10 \%$ & 1.357 & $6 \%$ & 1.645 & $0 \%$ \\
\hline
\end{tabular}

Axisymmetric simulations are made for the Apollo and Gemini entry vehicles to help determine whether the difference between the DSMC and windtunnel data is due to possible windtunnel wall boundary layer interaction with the larger diffuse shockwave structure incurred by the ballistic capsule shapes. If the Gemini windtunnel data agrees with DSMC data, then the Apollo simulation problem may be an isolated case. Figure 15 displays selected contour plots from the Apollo and Gemini axisymmetric simulations. These plots show that the upstream region contains sufficient free-stream to capture the diffuse shock structure near the vehicle, and that the peak translational temperature is not large enough to incur nitrogen dissociation.

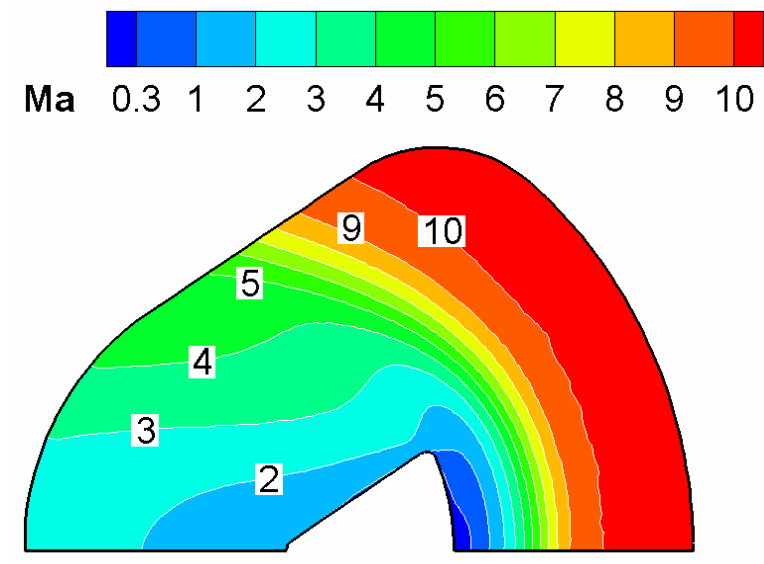

(a) Apollo Mach Number

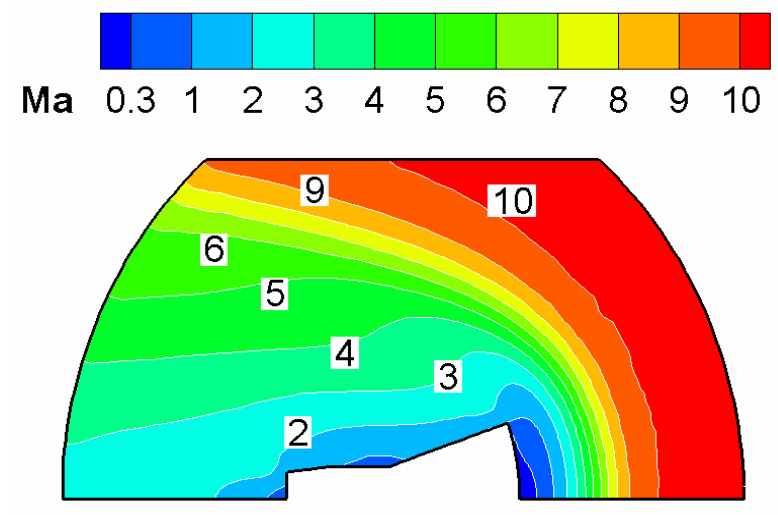

(b) Gemini Mach Number

Figure 15. Contour Plots of the Axisymmetric Simulations 


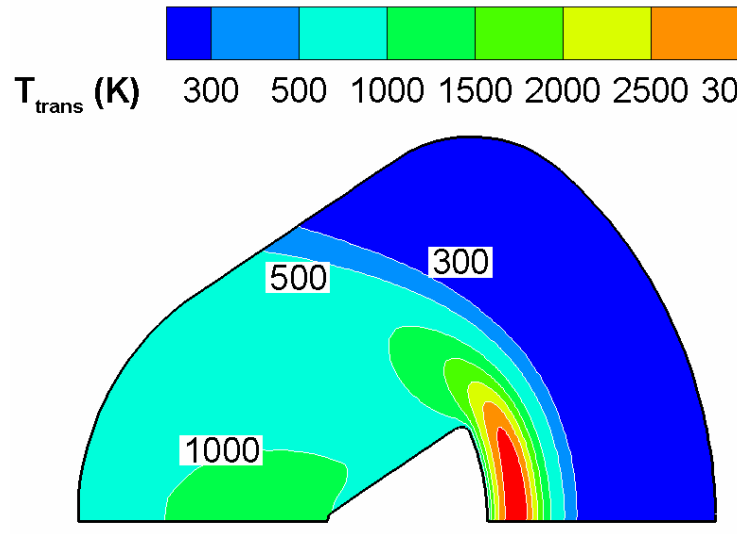

(c) Apollo Translational Temperature

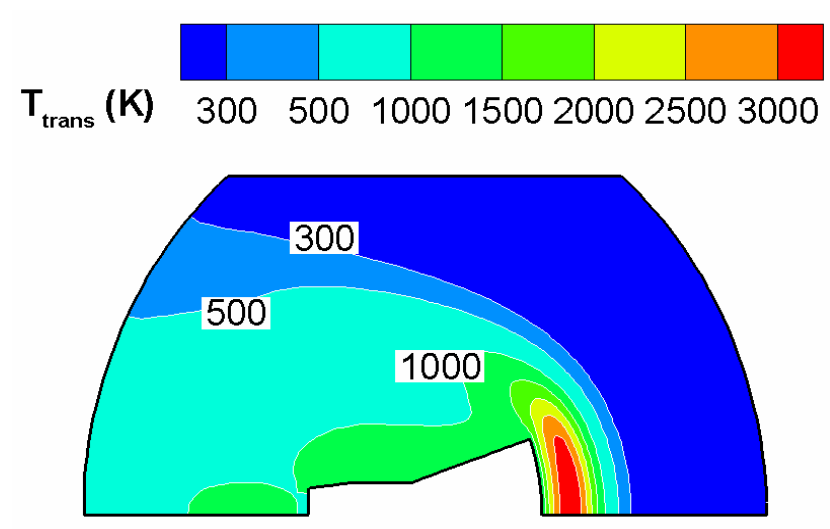

(d) Gemini Translational Temperature

\section{Figure 15. Continued.}

Table 5 provides comparisons of the windtunnel and axisymmetric simulation drag coefficients. A result from another DSMC code called DS2V, computed by Dr. Moss, is also listed, and again gives confidence in the MONACO procedures. For the ACM, the axisymmetric simulation value for $C_{D}$ is $1.2 \%$ larger than the threedimensional simulation value because of differences in grid cell types and cell distributions of the two and three dimensional meshes. Nevertheless, both results are about $12 \%$ smaller than the windtunnel result, shown in Fig. 14 (a) and listed in Table 5. The smaller diameter Gemini model is associated with a smaller disagreement in the drag coefficient, Table 5. The ballistic capsule shapes have larger disagreement between the DSMC and windtunnel data than the slender blunted-cone shape. The blunter shapes have larger diffuse shock structures that are more susceptible to interference with a growing windtunnel boundary layer. Thus, the windtunnel data for the blunter shapes may be inaccurate because of disregarded wall effects. Unfortunately, available reference ${ }^{20-22}$ do not provide sufficient details of the windtunnel geometry in order to adequately simulate the windtunnel test to accurately capture the growing wall boundary layer from the nozzle and determine the boundary layer effects on the windtunnel model aerodynamics.

Table 5 Axisymmetric Simulation Drag

\begin{tabular}{|l|rr|}
\hline Case & $\mathrm{C}_{\mathrm{D}}$ & Difference \\
\hline Apollo & & \\
Windtunnel & 1.85 & \\
MONACO & 1.65 & $-11.4 \%$ \\
DS2V & 1.66 & $-10.8 \%$ \\
& & \\
Gemini & & \\
Windtunnel & 1.86 & \\
MONACO & 1.72 & $-7.5 \%$ \\
\hline
\end{tabular}

\section{Conclusion}

Aerodynamic properties, drag, lift, pitching moment, and lift-to-drag ratio, of entry vehicle windtunnel test models within a hypersonic, near-rarefied nitrogen gas environment were analyzed using three-dimensional DSMC computations. Modified Newtonian and free molecular flow models were used to develop procedures to compute the aerodynamic properties from the DSMC analysis. For the blunted-cone windtunnel model, DSMC and windtunnel aerodynamic data agreed well, and a 15\% reduction in gas-surface thermal energy accommodation did not significantly affect the DSMC results. For the Apollo windtunnel test, DSMC and windtunnel data did not agree well. The drag from DSMC was roughly 13\% less than that from the windtunnel test. A second set of DSMC results, generated by a different code, DS2l3V, provided confidence in the MONACO procedures. Consequently, the possibility of erroneous Apollo windtunnel test results was examined. A sensitivity study demonstrated that the drag coefficient experienced insignificant changes when the reported Mach number or reservoir temperature was 
perturbed by $\pm 20 \%$. Therefore, the Mach number and reservoir temperature were considered to be reported accurately enough. Then, a Gemini windtunnel test simulation was performed to determine whether the problem was related to the blunt shape of the vehicle. The ballistic capsule shapes have larger disagreement between the DSMC and windtunnel data than the slender blunted-cone shape. The blunter shapes have larger diffuse shock structures that are more susceptible to interference with a growing windtunnel boundary layer. Unfortunately, available references $^{20-22}$ do not provide sufficient details of the windtunnel geometry in order to adequately simulate the windtunnel test to accurately capture the growing wall boundary layer from the nozzle and determine the boundary layer effects on the windtunnel model aerodynamics. From this study, it is recommended that more rarefied hypersonic windtunnel tests be conducted to broaden the existing literature, and that corresponding documentation be sufficiently detailed to enable comprehensive numerical simulation of the windtunnel tests.

\section{Acknowledgments}

This work is sponsored by the Space Vehicle Technology Institute, under NASA grant NCC-3989 with joint sponsorship from the Department of Defense. Computational resources on NASA's Columbia supercomputer are gratefully acknowledged. The authors gratefully acknowledge DS213V DSMC results provided by Dr. James Moss from NASA Langley Research Center.

\section{References}

${ }^{1}$ Boylan, D. E., and Potter, J. L., "Aerodynamics of Typical Lifting Bodies under Conditions Simulating Very High Altitudes," AIAA Journal, Vol. 5, 1967, pp.226-232.

${ }^{2}$ Griffith, B. J., "Comparison of Aerodynamic Data from the Gemini Flights and AEDC-VKF Wind Tunnels," Journal of Spacecraft, Vol. 4, No. 7, 1967, pp. 919-924.

${ }^{3}$ Griffith, B. J., and Boylan, D. E., "Reynolds and Mach Number Simulation of Apollo and Gemini Re-entry and Comparison with Flight," Specialists' Meeting on Hypersonic Boundary Layers and Flow Fields of the Fluid Dynamics Panel of AGARD, North Atlantic Treaty Organization, Paris, 1968, pp. 8-1-8-21.

${ }^{4}$ Boylan, D. E., and Griffith, B. J., "Simulation of the Apollo Command Module Aerodynamics at Re-entry Altitudes," Proceedings of the $3^{\text {rd }}$ National Conference on Aerospace Meteorology, American Meteorological Society, Boston, 1968, pp. 370-378.

${ }^{5}$ Bird, G. A., Molecular Gas Dynamics and the Direct Simulation of Gas Flows, Oxford University Press, 1994.

${ }^{6}$ Anderson, J. D., Hypersonic and High Temperature Gas Dynamics, McGraw-Hill Book Company, New York, 1989.

${ }^{7}$ Cajori, Florian, Sir Isaac Newton's Mathematical Principles of Natural Philosophy and His System of the World, University of California Press, Berkeley, 1934.

${ }^{8}$ Chandrasekhar, S., Newton's Principia for the Common Reader, Oxford University Press, New York, 1995.

${ }^{9}$ Gombosi, T. I., Gaskinetic Theory, Cambridge University Press, Cambridge, England, 1994.

${ }^{10}$ Sentman, L. H., "Free molecule flow theory and its application to the determination of aerodynamic forces," Lockheed Missile \& Space Co., LMSC-448514, 1961.

${ }^{11}$ Dietrich, S., and Boyd, I. D., "Scalar and Parallel Optimized Implementation of the Direct Simulation Monte Carlo Method," Journal of Computational Physics, Vol. 126, 1996, pp. 328-342.

${ }^{12}$ Koura, K., and Matsumoto, H., "Variable Soft Sphere Molecular Model for Air Species," Physics of Fluids, Vol. 4, 1992, pp. 1083-1085.

${ }^{13}$ Vijayakumar, P., Sun, Q., and Boyd, I. D., "Detailed Models of Vibrational-Translational Energy Exchange for the Direct Simulation Monte Carlo Method," Physics of Fluids, Vol. 11, 1999, pp. 2117-2126.

${ }^{14}$ Boyd, I. D., “Analysis of Rotational Nonequilibrium in Standing Shock Waves of Nitrogen,” AIAA Journal, Vol. 28, 1990, pp. 1997-1999.

${ }^{15}$ Lee, D. B., and Goodrich, W. D., "The Aerothermodynamic Environment of the Apollo Command Module During Superorbital Entry," NASA TN D-6792, 1972.

${ }^{16}$ Griffith, B. J., "Comparison of Data from the Gemini Flights and AEDC-VKF Wind Tunnels," AIAA Aerospace Sciences Meeting, AIAA, Washington, DC, 1967.

${ }^{17}$ Pro/ENGINEER Wildfire, Version 2.0, Parametric Technology Coorporation, Needham, MA, 2004.

${ }^{18}$ HyperMesh, Version 7.0, Altair Engineering Inc., Troy, MI, 2004.

${ }^{19}$ Moss, J. N., private communication, December 2005.

${ }^{20}$ Potter, J. L., Arney, G. D., Jr., Carden, W. H., and Kinslow, M., "Gasdynamic diagnosis of high-speed flows expanded from plasma states," IEEE Transactions on Nuclear Science, Vol. NS-11, No. 1, 1964, pp. 145-157.

${ }^{21}$ Potter, J. L., Arney, G. D., Jr., Carden, W. H., and Kinslow, M., "Irreversible flow in reservoir and throat sections of wind tunnels with constricted-arc heaters," Proceedings of AGARD Fluid Dynamics Panel Specialists Meeting, AGARDograph 84, Technical Editing and Reproduction Ltd., London, 1964, pp. 379-412

${ }^{22}$ Potter, J. L., Kinslow, M., Arney, G. D., Jr., and Bailey, A. B., "Initial results from a low-density hypersonic wind tunnel," Progress in Astronautics and Rocketry: Hypersonic Flow Research, edited by F. R. Riddell, Vol. 7, Academic Press Inc., New York, 1962, pp. 599-624. 